\title{
A Review of the Review Process
}

\author{
Leslie R. Dye, MD, FACMT
}

Associate Editor, Journal of Medical Toxicology

\section{Review:}

To view, look at, or look over again
To inspect, esp. formally or officially
To survey mentally; take a survey of
To discuss in a critical review; write a critical
report upon
To look back upon; view retrospectively
To present a survey of in speech or writing $[1]$

The word "review" seems so simple. Above is just a short list of the many definitions of the word. As an academic physician, I followed the classic path, including completing residency and fellowship, obtaining an academic appointment at a medical school, and publishing articles and textbook chapters. I served on the board of my professional organization, served on committees in the medical school, participated in annual scientific meetings, lectured nationally and internationally, taught medical students, residents, and fellows, gave Grand Rounds-oh well, you know the drill. A natural part of that process is to become a reviewer. My first reviews were of medical student and resident publications, but eventually I was asked to be a reviewer of a peer reviewed medical journal. But what were my qualifications? What is peer review, what is its purpose, how does it occur, has it been studied, and what are its implications?

According to the Uniform Requirements for Manuscripts Submitted to Biomedical Journals: Writing and Editing for Biomedical Publication, a peer review is an "[u]nbiased, independent, critical assessment [that] is an intrinsic part of all scholarly work, including the scientific process. Peer review is the critical assessment of manuscripts submitted to journals by experts who are not part of the editorial staff. Peer review can therefore be viewed as an important extension of the scientific process. Although its actual value has been little studied, and is widely debated, peer review helps editors decide which manuscripts are suitable for their journals, and helps authors and editors in their efforts to improve the quality of reporting. A peer reviewed journal is one that submits most of its published research articles for outside review. The number and kind of manuscripts sent for review, the number of reviewers, the reviewing procedures, and the use made of the reviewers' opinions may vary. In the interests of transparency, each journal should publicly disclose its policies in its instructions to authors" [2].

What is the purpose of peer review? There are a myriad of answers to this question. Maintaining objectivity, preserving solid science, avoiding conflict of interest, and preventing fraud are some of the obvious reasons. Peer reviewers assure the quality and suitability for publication in a journal, and reviews should be an educational process that allows the author to create better manuscripts by explaining problems about the paper to the author. The bottom line is that reviews insure quality manuscripts, and hence, improve the quality of the journal.

Until I became involved in the world of publishing and served in editorial positions in two fledgling medical journals, I really did not know the methods used to choose reviewers. I assumed that there was a complicated formula used and that it was the same for every journal. Both assumptions are false. When the Journal of Medical Toxicology (JMT) was started, there was a formal application to the ACMT Board for the position of Editorin-Chief and Associate Editor. With board oversight, those editors were responsible for creating an editorial board and a pool of qualified reviewers.

Because reviewing a medical journal submission takes an average of 2 hours and may take up to 20 hours, the compensation comes out to be a whopping ZILCH [3]. Journals with a large number of submissions may reject papers at the level of the editor and not send the paper through any additional review. Like any voluntary position, one of the first requirements for reviewers is willingness. For JMT, editors are assigned manuscripts and then the editors select reviewers. As would be expected, a reviewer is selected based on their expertise of the manuscript topic. The basis of this "expertise" is usually from the reviewers having written, researched, or spoken on the same or similar topic. With $J M T$ 's new electronic manuscript submission process (Editorial Manager), reviewers can be placed in a database that lists personal

Keywords: review, reviewers, peer review 
areas of expertise. The editor then matches the keywords in the paper with a reviewer's areas of expertise. Often an expert on the topic is known, but not yet in the electronic reviewer pool. In that case, the editor can invite an expert to review a manuscript and add the expert to the database.

In addition to the areas of expertise, the computer system also lists a summary of the reviewer's past performance, including how many reviews the peer-reviewer has done, the date the reviewer last reviewed, any papers currently being reviewed, and the time the reviewer takes to return a review. Quite a bit of detail is tracked for each reviewer and can also be accessed. The number of reviewers required for each submission varies with each journal. JMT requires two outside reviews prior to returning the submission to the editor. The editor then makes a decision; in addition, the editor can also supply additional comments for the author prior to making a decision. If a revision is required, the editor can choose to send the revision out for review again or make the decision without further review.

While being asked to review is based on known expertise, continuing to be asked depends on the reviewer's track record. Obviously, one criteria of a good reviewer is the submission of thoughtful reviews. The quality of reviews is difficult to quantify. Is a thoughtful review a basic scan of the manuscript to determine its value or is it a painstaking process that includes reanalysis of the data or even simple reconstruction of flawed sentences? Most agree that reviewer comments to the author are important, regardless of the ultimate disposition of the submission. A reviewer who consistently declines reviews, does not answer requests to review, or does not complete the review in a timely fashion may not be solicited to review in the future.

The review process has actually been studied to some extent, and it is an obviously imperfect science. While we would like to offer an objective, highly precise process, the fact that humans are involved makes perfect objectivity relatively impossible. Stephen Lock, when editor of the British Medical Journal (BMJ), evaluated a series of papers and determined which manuscripts he would publish before receiving any input from reviewers. After the full process of peer review, the outcome was similar to his original assessment [4].

As mentioned previously, reviewers may be rated using multiple factors; and according to Godlee and Jefferson, reviewers under the age of 40 years are better than those over 40 [5]. There are many possible reasons to explain this phenomenon. It may be that younger reviewers have more time (not always the case), are more current on reading the literature, or are more likely to try to please the editor with detailed information. Studies also suggest that $20 \%$ of reviews are outstanding, $20 \%$ are useless, and the rest are in between [2]. BMJ did a series of studies to determine how good peer review is at detecting errors in publications. In one study, they inserted major errors into all papers sent for review. No reviewer spotted all errors, and some did not spot any. In general, most reviewers detected $25 \%$ of the inserted errors [6]. Because trust is inherent, fraud is difficult to identify.
There is also a bias against studies with negative results. In general, if a treatment or intervention has no effect, many believe it is not important. But negative studies are important. The Lancet has agreed to consider protocols of studies that have yet to be performed. If the protocol is judged to be good and is followed, they guarantee publication of the paper, despite negative or positive results [7]. But this obviously requires even more commitment on the part of reviewers.

So, one may think that the review process could be improved if reviewers are given special training on the art of review. BMJ actually tested this hypothesis by randomizing reviewers into three groups: one group had no training, one received personal training in addition to a CD-ROM, and the third group received just the CD-ROM. After using a standardized instrument to measure the quality of reviews and counting the number of intentionally placed errors detected by reviewers, the results showed that training made no overall difference [8].

Most of us are aware, and some through personal experience, that if a paper is rejected by one journal, it is often submitted to another and eventually published. Even when changes suggested by reviewers are not done, publication ultimately occurs. According to Drummond Rennie, deputy editor of JAMA, there "seems to be no study too fragmented, no hypothesis too trivial, no literature citation too biased or too egotistical, no design too warped, no methodology too bungled, no presentation of results too inaccurate, too obscure, and too contradictory, no analysis too self-serving, no argument too circular, no conclusions too trifling or too unjustified, and no grammar and syntax too offensive for a paper to end up in print" [9].

In the long run, do reviewers have any influence other than slightly advancing or slowing an author's academic progress and lengthening an author's curriculum vitae? One only need look at the consequences of published articles to see the potential impact that medical journals have on influencing public opinion. Just look at the controversy regarding a link between immunizations and the development of autism. In 1998, Lancet published an article by Wakefield and others regarding the development of autism and an unusual bowel condition after receiving the MMR vaccination. While the study and an accompanying editorial admitted no proven link, the editorial did "warn" about the potential harm in vaccines [10]. The seemingly trivial statement may have had a serious impact. Many people are fearful of childhood immunizations, so much so that they will not have their children immunized. It is likely that the trend would have occurred even without this publication, but this demonstrates the potential impact and influence of medical journals on public health.

We already know that peer review is a very subjective process that is fraught with deficiencies and that the publication of scientific papers can have huge ramifications with regard to public health. But is there a better way? While the process is fairly subjective, for the most part, it is effective. It is unlikely that there are other viable alternatives at this point, but continued study of the process may improve quality. 


\section{REFERENCES}

1. www.dictionary.com

2. Smith R. The Trouble with Medical Journals. London: the Royal Society of Medicine Press; 2006. pp. 86.

3. International Committee of Medical Journal Editors [webpage on the Internet]. Uniform Requirements for Manuscripts Submitted to Biomedical Journals: Writing and Editing for Biomedical Publication [updated 2006, February]. Available from: http://www.icmje.org/index.html\#state

4. Lock S. A difficult balance: editorial peer review in medicine. London: Nufffield Provincials Hospital Trust, 1985.

5. Godlee F, Jefferson T, eds. Peer review in health sciences, $2^{\text {nd }}$ edition. London: BMJ Books, 2003.
6. Godlee F, Gale CR, Martyn CN. Effect on the quality of peer review of blinding reviewers and asking them to sign their reports: a randomized controlled trial. JAMA.

1998;280:237-240.

7. Horton R. Pardonable revisions and protocol reviews. Lancet. 1997;349:6.

8. Schroter S, Black N, Evans S, et al. Effects of training on quality of peer review. A randomized trial. BMJ.

2004;328:673.

9. Rennie D. Guarding the guardians: a conference on editorial peer review. JAMA. 1986;256:2391-2392.

10. Chen RT, DeStefano F. Vaccine adverse events: causal or coincidental? Lancet. 1998;351:611-612.

\section{ERRATUM}

A disclaimer was omitted from the article entitled, "Pediatric Sex Group Differences in Location of Snakebite Injuries Requiring Antivenom Therapy," by Michael J. Matteucci, MD, et al. (Volume 3, Number 3:103-106) of JMT. The disclaimer should read:

"The views expressed in this manuscript are those of the authors and do not necessarily reflect the official policy or position of the Department of the Navy, Department of Defense, nor the U.S. Government." 